\title{
Recent advances in the path toward the cure for chronic myeloid leukemia
}

\author{
Dong-Wook Kim \\ Department of Hematology, Seoul St. Mary's Hospital, The Catholic University of Korea, Seoul, Korea
}

p-ISSN 1738-7949 / e-ISSN 2092-9129 http://dx.doi.org/10.5045/kjh.2011.46.3.169

Korean J Hematol 2011;46:169-74.

Received on September 1, 2011 Accepted on September 9, 2011
Through the phase 3 International Randomized Study of Interferon vs. STI571 (IRIS) trial, imatinib emerged as the standard treatment for chronic myeloid leukemia $(\mathrm{CML})$ and has successfully prolonged the duration of both the chronic phase $(\mathrm{CP})$ and the disease-free state. The majority of newly diagnosed patients treated for CP-CML achieve a complete cytogenetic response (CCyR), and over time, most of these eventually achieve major molecular responses (MMRs) and even complete molecular responses (CMRs). In ongoing phase 3 randomized trials of second-generation tyrosine kinase inhibitors (TKIs), nilotinib and dasatinib have been found to have superior efficacies in helping achieve cytogenetic and molecular responses, including MMRs and CMRs. However, only the MMR rate was significantly higher in bosutinib compared with the imatinib control, but not in CCyR rate. Current reports of imatinib discontinuation suggested that achieving CMR is an important prerequisite for CML to be cured. Recent data from the STIM (Stop Imatinib) trial showed that imatinib can be successfully discontinued in patients who achieve a certain level of CMR. Standardized real-time quantitative reverse transcriptasepolymerase chain reaction (RQ-PCR) assays have been available in routine clinical practice, and efforts are being focused on achieving higher sensitivity and optimizing the time of imatinib discontinuation. Although very few patients are cured by administration of only Bcr-Abl TKIs, including imatinib and second-generation TKIs, current advances may eventually make this possible. This report summarizes the detailed clinical data obtained in the DASISION, ENESTnd, and BELA studies and discusses high-sensitivity detection methods and future therapeutic strategies.

Key Words CML, Imatinib, Nilotinib, Dasatinib, PCR, Leukemia stem cell

\section{LONG-TERM MOLECULAR RESPONSE OF IMATINIB: DATA FROM IRIS AND HAMMERSMITH AND SEOUL ST. MARY'S HOSPITAL}

\section{Incidence of molecular responses}

In the IRIS trial, previously untreated CP-CML patients $(\mathrm{N}=1,106)$ were randomly assigned to receive either imatinib $(\mathrm{N}=553)$ or interferon (IFN)- $\alpha$ plus cytarabine $(\mathrm{N}=553)$. During the sixth year of study treatment, the cumulative best CCyR rate was $82 \%$. The estimated event-free survival at 6 years was 83\%, and the estimated progression-free survival (PFS) rate was 93\% [1]. The IRIS study showed that during the median follow-up of 19 months, $21 \%$ and $4 \%$ of patients in the imatinib group had achieved MMR and CMR, respectively [2]. In a substudy of 53 imatinib-treated IRIS patients, the probability of Bcr-Abl being undetectable increased considerably from 36 to 81 months of first-line imatinib treatment ( $7 \%$ vs. $52 \%)$. Bcr-Abl became undetectable in 18 of 53 patients, and none of whom lost MMR after a median follow-up of 33 months. In contrast, MMR was lost in 6 of $22(27 \%)$ patients with sustained detectable Bcr-Abl [3]. In a study conducted in the Hammersmith hospital on $204 \mathrm{CP}-\mathrm{CML}$ patients receiving imatinib as first-line therapy, at 5 years, 159 patients (77\%) had achieved a CCyR (median time, 7 months; range, 3-55.4 months), 80 (39\%) had achieved an MMR (median time, 15.7 months; range, 2-73 months), and 10 (5\%) had achieved a CMR (median time, 30.7 months; range, 12-67.4 months). The cumulative incidences of CCyR and MMR were $82.7 \%$ and $50.1 \%$, respectively. Of the 80 patients who achieved an MMR, the response was sustained in $90 \%$ [4]. In our current study with 363 new CP patients, the cumulative incidences of CCyR, MMR, and $\mathrm{CMR}^{4.5}$ at 5 years increased to $92 \%, 52 \%$, and $11 \%$, respectively. At 7 years, the cumulative incidences of CCyR, MMR, and $\mathrm{CMR}^{4.5}$ were $94 \%, 60 \%$, and $14 \%$

This is an Open Access article distributed under the terms of the Creative Commons Attribution Non-Commercial License (http://creativecommons.org/licenses/by-nc/3.0) which permits unrestricted non-commercial use, distribution, and reproduction in any medium, provided the original work is properly cited. 
respectively. In addition, when compared to the group receiving less than $400 \mathrm{mg} / \mathrm{d}$, the $400 \mathrm{mg} / \mathrm{d}$ group demonstrated a better 6-year cumulative incidence of MMR (82\% vs. $66 \%$, respectively, $P=0.014)$ and $\mathrm{CMR}^{4.5}$ (38\% vs. $13 \%$, respectively, $P=0.0042$ ) (unpublished data). Therefore, response rates might be directly correlated with treatment intensity.

\section{Significance of molecular responses}

Several studies have reported different clinical implications of MMRs at specific time points in long-term survival outcomes. The IRIS study proved that MMRs at 12 and 18 months can predict significantly better PFS [2]. Moreover, the achievement of an MMR by 18 months of therapy was proposed to be the goal of therapy [5]. However, other studies have reported that such differences may not be clinically relevant and that only the achievement of CCyR was significant in order to predict survival $[4,6]$. Early achievement of molecular responses correlates with achievement of MMRs and a reduction in the number of events. As summarized in Table 1, the prognostic significance of early molecular responses is clear; patients with a Bcr-Abl ratio of $1 \%$ or less at 3 months had very low event rates, with a median follow-up of 53 months [7]. The CMR rate gradually increased with continuing imatinib treatment, and CMR was more common in patients who achieved an MMR early.

\section{MOLECULAR RESPONSES IN ENESTnd, DASISION,} AND BELA TRIALS

\section{Frontline second-generation TKI studies; ENESTnd,} DASISION, and BELA

Investigations are currently underway to determine the efficacy and safety of second-generation Bcr-Abl TKIs in the treatment of newly diagnosed CP-CML, including the multicenter, phase 3, randomized clinical trials "Evaluating Nilotinib Efficacy and Safety in Clinical Trials-Newly Diagnosed Patients" (ENESTnd) [8] and "Dasatinib versus Imatinib Study in Treatment-Naïve CML Patients"
(DASISION) [9]. These studies have shown that Nilotinib and dasatinib have superior efficacies than imatinib. Bosutinib, currently an investigational agent not yet approved for CML treatment, is also being studied in the "Bosutinib versus Imatinib in Patients with Chronic Phase Chronic Myeloid Leukemia" (BELA) trial [10]. In the ENESTnd study, 846 patients were randomly assigned to receive $300 \mathrm{mg}$ nilotinib twice daily ( $\mathrm{N}=282), 400 \mathrm{mg}$ nilotinib twice daily ( $\mathrm{N}=281$ ), and $400 \mathrm{mg}$ imatinib once daily (QD; N=283). In the DASISION trial, 519 patients with newly diagnosed CP-CML were randomized to receive first-line treatment with either $100 \mathrm{mg}$ dasatinib QD ( $\mathrm{N}=259)$ or 400 mg imatinib QD ( $=260)$. In the BELA study, 502 patients with newly diagnosed CP-CML were randomized to receive first-line treatment with either $500 \mathrm{mg}$ bosutinib QD ( $\mathrm{N}=250)$ or $400 \mathrm{mg}$ imatinib QD ( $\mathrm{N}=252)$. The ENESTnd, DASISION, and BELA trials had different study designs, primary endpoints, and definitions. Therefore, direct comparison of the results is not possible. Presently, 24 months of data from the ENESTnd and DASISION studies, and 18 months of data from the BELA study are available, and significantly higher rates of MMR and $\mathrm{CMR}^{4.5}$ were observed with nilotinib, dasatinib, and bosutinib than with imatinib [10-12].

2. Comparison of molecular responses between secondgeneration TKIs and Imatinib

By 24 months into the ENESTnd study, significantly more patients had achieved an MMR with nilotinib than with imatinib: $71 \%$ of patients receiving nilotinib at $300 \mathrm{mg}$ twice daily, $67 \%$ of patients receiving nilotinib at $400 \mathrm{mg}$ twice daily, and $44 \%$ of patients receiving imatinib showed an MMR ( $P<0.0001$ for both comparisons). In addition, significantly more patients in the nilotinib groups achieved a $\mathrm{CMR}^{4.5}$ at any time than those in the imatinib group $(26 \%$ of patients receiving nilotinib at $300 \mathrm{mg}$ twice daily, $21 \%$ of patients receiving nilotinib at $400 \mathrm{mg}$ twice daily, and $10 \%$ of patients receiving imatinib achieved a CMR) [11]. By 24 months into the DASISON study, the rate of MMRs was superior for dasatinib vs. imatinib ( $64 \%$ vs. $46 \%$, respectively), and the $\mathrm{CMR}^{4.5}$ rate was also higher for dasatinib vs. imatinib ( $17 \%$ vs. $8 \%$, respectively) [12]. From 12 to

Table 1. Relationship between early molecular response and patient outcomes.

\begin{tabular}{|c|c|c|c|c|c|c|}
\hline \multirow{3}{*}{$\begin{array}{l}\text { Bcr-Abl 1/ABL1 } \\
\text { transcript ratio }\end{array}$} & \multicolumn{6}{|c|}{ Probability of outcome according to transcript ratio at specified time points, $\%$} \\
\hline & \multicolumn{3}{|c|}{ MMR (Bcr-Abl 1/ABL1<0.05\%) } & \multicolumn{3}{|c|}{ Event $^{a}$} \\
\hline & 3 months & 6 months & 12 months & 3 months & 6 months & 12 months \\
\hline$\leq 0.1 \%$ & 100 & 96 & 97 & 4 & 1 & 3 \\
\hline$>0.1 \%$ to $1 \%$ & 84 & 69 & 61 & 3 & 7 & 2 \\
\hline$>1 \%$ to $10 \%$ & 53 & 44 & 20 & 11 & 9 & 8 \\
\hline$>10 \%$ & 33 & 15 & 7 & 13 & 23 & 50 \\
\hline
\end{tabular}


24 months, dasatinib also continued to show better efficacy than imatinib for the treatment of CML. The MMR rate at 18 months in the BELA study was higher with bosutinib (46\%; 95\% CI, 39-52\%) than with imatinib (38\%; 95\% CI, $32-44 \%$ ), and $9 \%$ of patients achieved a $\mathrm{CMR}^{4.5}$ with bosutinib, compared with $4 \%$ who achieved a CMR with imatinib [10]. Overall, patients achieved their first molecular responses faster and deeper with all 3 of these second-generation TKIs than with imatinib.

\section{STUDIES ON DISCONTINUATION OF TKIs}

\section{Discontinuation of imatinib}

Although imatinib therapy is effective and currently considered a front-line therapy in patients with CML, it is still unclear whether intermittent imatinib therapy can be safely employed in particular situations such as pregnancy and serious adverse events. Thus far, in studies on the discontinuation of TKI therapy, $100 \%$ of patients with less than a CMR have relapsed (Table 2) [13-15]. Therefore, it may be necessary for patients to achieve a CMR before physicians consider potentially discontinuing TKI therapy [16]. In our study, the majority of patients maintained their best achieved response after resuming imatinib treatment [13]. Therefore, although imatinib cannot be discontinued completely, transient interruption can be considered for the treatment of patients with CML in particular situations such as serious or life-threatening concomitant diseases, pregnancy, or major surgery.

In the STIM study, patients with more than 2 years of documented CMRs while on imatinib therapy attempted therapy discontinuation. A total of 69 patients were followed up for more than 12 months. Nineteen of 34 patients (55.9\%) who received prior INF- $\alpha$ therapy relapsed, and 23 of 35 patients $(65.7 \%)$ who received imatinib as first-line therapy relapsed. The Sokal scores showed that 17 of 35 patients (48.6\%), 15 of 23 patients (65.2\%), and 7 of 8 patients $(87.5 \%)$ who relapsed were at low, intermediate, or high risk, respectively. In addition, 27 of 51 patients (52.9\%) who received more than 50 months of imatinib treatment relapsed, compared with 15 of 18 patients (83.3\%) who received less than 50 months of imatinib therapy [16]. Typically,

Table 2. Results of imatinib discontinuation in different clinical settings.

\begin{tabular}{lc}
\hline $\begin{array}{c}\text { Response at time of treatment } \\
\text { discontinuation }\end{array}$ & $\begin{array}{c}\text { Patients with molecular and/or } \\
\text { cytogenetic relapse, } \%\end{array}$ \\
\hline $\mathrm{CCyR}^{13}$ & $100 \%$ \\
$\mathrm{MMR}^{14}$ & $100 \%$ \\
$\mathrm{MMR}^{14}$ CCyR, MCyR & $100 \%$ \\
$\mathrm{CMR}^{15}$ for $\geq 2$ years on imatinib & 16 \\
\hline
\end{tabular}

Abbreviations: CCyR, complete cytogenetic response; MMR, major molecular response; CMR, complete molecular response. patients who enrolled in the STIM trial had deeper and longer molecular responses while on imatinib therapy than patients in other studies, and therefore, discontinuation of therapy should not be attempted outside of a controlled clinical trial with frequent monitoring.

\section{Discontinuation of second-generation TKIs}

In a study on discontinuation of dasatinib or nilotinib in 17 adult patients who had achieved a $\mathrm{CMR}^{4.5}$ [17], of the 12 patients with a minimum follow-up period of 6 months (median, 12; range, 7-18), 11 had imatinib intolerance and 1 had imatinib resistance, and 30\% (4/12) of patients lost the MMR by 6 months of discontinuation. The MMR was rapidly regained upon re-introduction of early second-generation TKIs. Treatment was also restarted in 1 patient in whom there was no MMR loss but who showed CMR loss on 2 consecutive assessments. Seven patients remained off therapy at the last follow-up after a median of 11 months (range, 7-18), with either a stable CMR or weakly detectable Bcr-Abl transcripts on one or more occasions. Although a longer follow-up with more patients will be required, the study provided a basis for further studies on discontinuation of second-generation TKIs. In addition, since current frontline second-generation TKI data from the DASISION and ENESTnd studies are showing that the probability of persistent CMR by 36 months of follow-up remains higher with dasatinib and nilotinib than with imatinib, more patients receiving second-generation TKI therapies may be safely discontinued in the future.

\section{HIGH-SENSITIVITY PCR TECHNOLOGY}

\section{Definition of molecular responses by conventional RQ- PCR}

The principal means of monitoring a patient's response to therapy is to assess their hematologic, cytogenetic, and molecular responses as well as their long-term outcomes [5, 18]. Since therapies for CML have improved, patients have been able to achieve responses at the molecular level. Therefore, more sensitive measurements are necessary to detect minimal residual disease (MRD). Real-time quantitative polymerase chain reaction (RQ-PCR) assessment can detect deeper levels of response, up to a 5-log reduction [6]. Generally, molecular response is a measure of the level of Bcr-Abl transcripts as determined by RQ-PCR and is described as a ratio of $\mathrm{Bcr}-\mathrm{Abl}$ to a housekeeping gene (such as $A B L, B C R$, or GUS). An MMR is achieved when a patient reaches a 3-log reduction in Bcr-Abl transcripts from a baseline value defined in the IRIS trial [2, 19]. Since inter-laboratory variation in the methods of conducting RQ-PCR makes comparison of data between laboratories difficult, the international scale (IS) has been developed to allow for cross-laboratory comparison of PCR results [20]. The IS is defined by 2 values: (1) a baseline value (i.e., pretreatment with imatinib) of the Bcr-Abl/ABL ratio, which was defined based on an analysis of baseline levels in patients from the IRIS 
trial (100\%), and (2) a 3-log reduction thereof, or a reduction to $0.1 \%$ or less, which is defined as an MMR [2]. To convert the PCR results obtained in a laboratory to the IS, the results are modified using a laboratory-specific conversion factor that is calculated by comparing patient samples against a reference method [20]. Deeper molecular responses are now defined as achieving a $\mathrm{CMR}^{4.0}$ of more than 4.0 -log reduction $\left(\mathrm{Bcr}-\mathrm{Abl}^{\mathrm{IS}} \leq 0.01 \%\right.$ ), a $\mathrm{CMR}^{4.5}$ of more than 4.5 -log reduction $\left(\mathrm{Bcr}_{-} \mathrm{Abl}^{\mathrm{IS}} \leq 0.005 \%\right)$, and a $\mathrm{CMR}^{5.0}$ of more than 5-log reduction in Bcr-Abl transcripts (Bcr-Abl ${ }^{\mathrm{IS}} \leq 0.001 \%$ ) [21, 22]. In the STIM trial, the selection criteria for candidates was based on the duration of imatinib therapy and the duration of PCR negativity prior to discontinuation, and conventional RQ-PCR was employed to measure PCR negativity. However, the fact that the absolute number of residual leukemia clones could not be measured under the detection limits of conventional RQ-PCR might have resulted in relapse in more than half of the patients. This indicates that conclusions cannot be drawn about whether a patient could safely discontinue therapy solely based on conventional RQ-PCR. The absence of Bcr-Abl transcripts in conventional RQ-PCR is sometimes unreliable since some patients, despite being PCR-negative, relapse and PCR negativity may be maintained after imatinib discontinuation [23, 24]. It might be necessary to develop more sensitive assays that can provide a more reliable estimation of the therapeutic response of the patient, and thus allow further classification of patients who may discontinue imatinib without the risk of relapse. With the advent of more potent TKIs, more sensitive assays would also be able to extend the period of measurable disease by several years in most patients with CML. This will help in further assessment of the kinetics of Bcr-Abl under the detection limit of conventional RQ-PCR, whereas conventional RQ-PCR will become less useful for many patients who show dramatic responses to therapy and in whom Bcr-Abl transcripts in undetectable.

\section{High-sensitivity PCR technologies}

With the emergence of increasingly efficacious therapies leading up to imatinib, the majority of patients with $\mathrm{Ph}+$ CML were able to achieve deeper levels of response. Increasingly sophisticated and sensitive methods of disease detection have been developed in parallel to facilitate the detection of these deeper patient responses. To demonstrate the feasibility of more sensitive approaches, several methods have been developed using DNA-based PCR [25] and RNA-based PCR assays [26]. Replicated PCR (rRQ-PCR), DNA-based PCR, and RNA-based digital PCR (dPCR) assays have successfully detected $\mathrm{Bcr}-\mathrm{Abl}$ transcripts that were previously not detectable by conventional RQ-PCR, and these data show the potential feasibility of high-sensitivity PCR approaches for molecular monitoring and their clinical relevance in future strategies for drug discontinuation by allowing further characterization of patients who achieve PCR negativity in conventional RQ-PCR assays. In our study, rRQ-PCR allowed for a 2-log improvement in detection sensitivity, and therefore, use of this method might increase the chances of detecting very low Bcr-Abl transcript levels, the probability of which increases with the number of repetitions [26]. In addition to rRQ-PCR, the concept of partitioning in $\mathrm{dPCR}$, in conjunction with a pre-amplification step, also successfully achieved a 2-3-log improvement in detection sensitivity. Although implementation of a pre-amplification step prior to conventional RQ-PCR also increased the sensitivity to a level comparable to that of rRQ-PCR, significant variations in the detection of Bcr-Abl copy numbers were evident in samples with low levels of Bcr-Abl transcripts in replicate experiments. Therefore, although the sensitivity was improved by pre-amplification in conventional RQ-PCR, dPCR would be an attractive alternative tool for accurate detection of low levels of MRD with high sensitivity. In the future, more strict PCR sensitivity criteria using highly sensitive technologies should be employed to accurately assess Bcr-Abl transcript levels prior to discontinuation and then to consider whether discontinuation of TKI therapy may be safe.

\section{THERAPEUTIC STRATEGIES FOR POTENTIAL CURE}

\section{Definition of curability}

In practical terms, some criteria have defined curability as (1) sustained molecular response, (2) successful discontinuation of TKI therapy, (3) 100\% CML-related survival, and (4) quality of life (QoL) comparable to that of age-matched population. Although considerable progress has recently been made in CML therapy, a true cure for the disease can only be obtained by our present therapeutic means in a rather small minority of patients. Since leukemia stem cells (LSCs) in CML are insensitive to imatinib treatment [27], and the self-renewal ability of LSCs can result in re-emergence of disease even after a long period of RQ-PCR negativity, sustained undetectable MRD is obtained only in a minority of CML patients treated with TKIs. It still is not clear whether these patients are definitively cured of leukemia, or whether LSCs persist in their bone marrow. The relationship between LSC persistence and the potential risk of disease relapse for patients with long-term undetectable MRD warrants further investigation [28].

\section{New therapeutic targets for curing CML}

Although the molecular differences in Bcr-Abl expression between hematopoietic stem cells (HSCs) and LSCs are still unclear, the best means of identifying stem cell targets in CML is to fully understand CML-initiating genetic changes. Recently, several new targets or drugs have been found to inhibit LSCs in cultured human CD34+ CML cells or in mouse models of Bcr-Abl-induced CML, including an Alox5 pathway inhibitor, Hsp90 inhibitors, omacetaxine, a hedgehog inhibitor, and the farnesyl transferase inhibitor BMS-214662. To obtain the ultimate goal of a cure for CML, 2 strategies have been tested. One important strategy has been to inhibit target genes such as those encoding hedgehog [29], Wnt/ $\beta$-catenin [30], and Bim-1 [31] in order to function- 


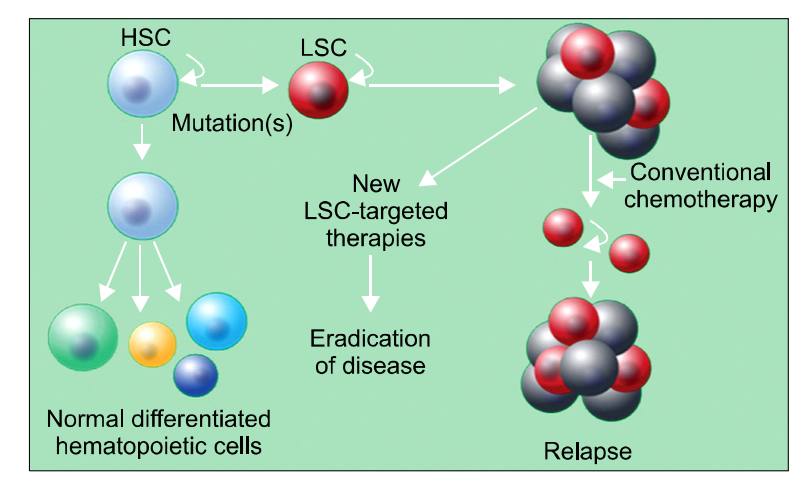

Fig. 1. Role of normal stem cells and leukemia stem cells.

ally regulate both normal stem cells and LSCs. Another approach has been the specific targeting of LSC-related genes, but not those of normal stem cells, for developing new anticancer therapies in the future (Fig. 1) [32].

Currently, several drugs targeting LSCs have been reported. The hedgehog pathway is active in many tissues, where it plays a critical role in hematopoiesis and is activated in $\mathrm{Ph}+\mathrm{CML}$ stem cells [33]. The smoothened antagonist, LDE225, is currently in phase 1 clinical trial for the treatment of $\mathrm{Ph}+\mathrm{CML}$ and other cancers. Janus kinase 2 inhibition may inhibit stem cells [34], and INF may stimulate quiescent LSCs to proliferate, making them susceptible to TKI therapy [35]. Residual disease may be the result of the presence of LSCs, which can act as a reservoir of disease that cannot be eradicated by TKI therapy alone [27, 36]. Relapses can occur even in patients who have undergone potentially curative stem cell transplants, suggesting that residual disease may be present even in patients with prolonged CMRs [37, 38]. Therefore, the inhibition of LSCs may be a crucial step in curing CML. Several trials utilizing INF maintenance therapies and/or combination therapies with potent TKIs and/or LSC-targeting agents are ongoing. Future CML management might include earlier use of these agents to help more patients achieve CMRs and may be a means of curing CML.

\section{CONCLUSION}

During the past decade, considerable progress has been made in understanding the biology of CML, which has raised hopes that this disease may be curable. Early intensification using combination therapies with more potent TKI- and LSC-targeted agents is required for the development of more sensitive PCR assays. The achievement of deeper responses, measured only by highly sensitive assays, will establish a necessary first step toward potentially discontinuing drug therapy.

\section{REFERENCES}

1. Hochhaus A, O'Brien SG, Guilhot F, et al. Six-year follow-up of patients receiving imatinib for the first-line treatment of chronic myeloid leukemia. Leukemia 2009;23:1054-61.

2. Hughes TP, Kaeda J, Branford S, et al. Frequency of major molecular responses to imatinib or interferon alfa plus cytarabine in newly diagnosed chronic myeloid leukemia. N Engl J Med 2003;349:1423-32.

3. Branford S, Seymour JF, Grigg A, et al. BCR-ABL messenger RNA levels continue to decline in patients with chronic phase chronic myeloid leukemia treated with imatinib for more than 5 years and approximately half of all first-line treated patients have stable undetectable BCR-ABL using strict sensitivity criteria. Clin Cancer Res 2007;13:7080-5.

4. de Lavallade H, Apperley JF, Khorashad JS, et al. Imatinib for newly diagnosed patients with chronic myeloid leukemia: incidence of sustained responses in an intention-to-treat analysis. J Clin Oncol 2008;26:3358-63.

5. Baccarani M, Cortes J, Pane F, et al. Chronic myeloid leukemia: an update of concepts and management recommendations of European LeukemiaNet. J Clin Oncol 2009;27:6041-51.

6. Kantarjian H, O'Brien S, Shan J, et al. Cytogenetic and molecular responses and outcome in chronic myelogenous leukemia: need for new response definitions? Cancer 2008;112:837-45.

7. Quintás-Cardama A, Kantarjian H, Jones D, et al. Delayed achievement of cytogenetic and molecular response is associated with increased risk of progression among patients with chronic myeloid leukemia in early chronic phase receiving high-dose or standard-dose imatinib therapy. Blood 2009;113:6315-21.

8. Saglio G, Kim DW, Issaragrisil S, et al. Nilotinib versus imatinib for newly diagnosed chronic myeloid leukemia. N Engl J Med 2010;362:2251-9.

9. Kantarjian H, Shah NP, Hochhaus A, et al. Dasatinib versus imatinib in newly diagnosed chronic-phase chronic myeloid leukemia. N Engl J Med 2010;362:2260-70.

10. Brümmendorf T, Gambacorti-Passerini C, Cortes J, et al. The BELA trial: bosutinib versus imatinib in patients with chronic phase chronic myeloid leukemia; 18-month follow-up. Haematologica 2011;96(Suppl 2):204(abst 0485).

11. Kantarjian HM, Hochhaus A, Saglio G, et al. Nilotinib versus imatinib for the treatment of patients with newly diagnosed chronic phase, Philadelphia chromosome-positive, chronic myeloid leukaemia: 24-month minimum follow-up of the phase 3 randomised ENESTnd trial. Lancet Oncol 2011;12:841-51.

12. Hochhaus A, Shah N, Cortes J, et al. Efficacy and safety of dasatinib compared with imatinib in patients with newly diagnosed chronic myeloid leukemia in chronic phase (CML-CP): minimum 24-month follow-up from the DASISION trial. Haematologica 2011;96(Suppl 2):422(abst 1011).

13. Goh HG, Kim YJ, Kim DW, et al. Previous best responses can be re-achieved by resumption after imatinib discontinuation in patients with chronic myeloid leukemia: implication for intermittent imatinib therapy. Leuk Lymphoma 2009;50:944-51.

14. Koskenvesa P, Mustjoki S, Rasanen A, et al. Imatinib discontinua- 
tion following a major molecular response: impact of interferon alpha and leukemia stem cell burden (The STOP Study). Blood 2008;112:738(abst 2121).

15. Kuwabara A, Babb A, Ibrahim A, et al. Poor outcome after reintroduction of imatinib in patients with chronic myeloid leukemia who interrupt therapy on account of pregnancy without having achieved an optimal response. Blood 2010;116:1014-6.

16. Mahon FX, Réa D, Guilhot J, et al. Discontinuation of imatinib in patients with chronic myeloid leukaemia who have maintained complete molecular remission for at least 2 years: the prospective, multicentre Stop Imatinib (STIM) trial. Lancet Oncol 2010;11: 1029-35.

17. Rea D, Rousselot P, Nicolini F, et al. Cessation of dasatinib or nilotinib therapy in chronic-phase chronic myeloid leukaemia patients with sustained complete molecular responses. Haematologica 2011;96(Suppl 2):423-4(abst 1014).

18. Baccarani M, Saglio G, Goldman J, et al. Evolving concepts in the management of chronic myeloid leukemia: recommendations from an expert panel on behalf of the European LeukemiaNet. Blood 2006;108:1809-20.

19. Hughes T, Deininger M, Hochhaus A, et al. Monitoring CML patients responding to treatment with tyrosine kinase inhibitors: review and recommendations for harmonizing current methodology for detecting BCR-ABL transcripts and kinase domain mutations and for expressing results. Blood 2006;108: 28-37.

20. Branford S, Fletcher L, Cross NC, et al. Desirable performance characteristics for BCR-ABL measurement on an international reporting scale to allow consistent interpretation of individual patient response and comparison of response rates between clinical trials. Blood 2008;112:3330-8.

21. Hughes TP, Hochhaus A, Saglio G, et al. ENESTnd update: continued superiority of nilotinib versus imatinib in patients with newly diagnosed chronic myeloid leukemia in chronic phase (CML-CP). Blood 2010;116:94-5(abst 207).

22. Choi SY, Goh HG, Kim D, et al. Dynamics of molecular response to standard-dose imatinib in new CP chronic myeloid leukemia patients after achieving CMR4.0. Haematologica 2011;96(Suppl 2):62(abst 0153).

23. Sawyers CL. Chronic myeloid leukemia. N Engl J Med 1999;340:1330-40.

24. Mauro MJ, Druker BJ, Maziarz RT. Divergent clinical outcome in two CML patients who discontinued imatinib therapy after achieving a molecular remission. Leuk Res 2004;28(Suppl 1): S71-3.

25. Ross DM, Branford S, Seymour JF, et al. Patients with chronic myeloid leukemia who maintain a complete molecular response after stopping imatinib treatment have evidence of persistent leukemia by DNA PCR. Leukemia 2010;24:1719-24.

26. Goh HG, Lin M, Fukushima T, et al. Sensitive quantitation of minimal residual disease in chronic myeloid leukemia using nanofluidic digital polymerase chain reaction assay. Leuk Lymphoma 2011;52:896-904.

27. Graham SM, Jørgensen HG, Allan E, et al. Primitive, quiescent, Philadelphia-positive stem cells from patients with chronic myeloid leukemia are insensitive to STI571 in vitro. Blood 2002;99:319-25.

28. Chomel JC, Bonnet ML, Sorel N, et al. Leukemic stem cell persistency in chronic myeloid leukemia patients with sustained undetectable molecular residual disease. Blood 2011 [Epub ahead of print].

29. Dierks C, Beigi R, Guo GR, et al. Expansion of Bcr-Abl-positive leukemic stem cells is dependent on Hedgehog pathway activation. Cancer Cell 2008;14:238-49.

30. Zhao C, Blum J, Chen A, et al. Loss of beta-catenin impairs the renewal of normal and CML stem cells in vivo. Cancer Cell 2007;12:528-41.

31. Lessard J, Sauvageau G. Bmi-1 determines the proliferative capacity of normal and leukaemic stem cells. Nature 2003;423: 255-60.

32. Jamieson CH, Ailles LE, Dylla SJ, et al. Granulocyte-macrophage progenitors as candidate leukemic stem cells in blast-crisis CML. N Engl J Med 2004;351:657-67.

33. Medina V, Calvo MB, Díaz-Prado S, Espada J. Hedgehog signalling as a target in cancer stem cells. Clin Transl Oncol 2009;11:199-207.

34. Kuroda J, Taniwaki M. Life and death of leukemic cells under Bcr-Abl signaling control. Curr Cancer Ther Rev 2009;5:303-9.

35. Essers MA, Offner S, Blanco-Bose WE, et al. IFNalpha activates dormant haematopoietic stem cells in vivo. Nature 2009;458: 904-8.

36. Jørgensen HG, Allan EK, Jordanides NE, Mountford JC, Holyoake TL. Nilotinib exerts equipotent antiproliferative effects to imatinib and does not induce apoptosis in CD34+ CML cells. Blood 2007;109:4016-9.

37. Akahane D, Ito Y, Sumi M, Tauchi T, Kimura Y, Ohyashiki K. Relapse of chronic myeloid leukemia-chronic phase 14 years after allogeneic hematopoietic stem cell transplantation. Int J Hematol 2008;88:119-20.

38. Robin M, Guardiola P, Devergie A, et al. A 10-year median follow-up study after allogeneic stem cell transplantation for chronic myeloid leukemia in chronic phase from HLA-identical sibling donors. Leukemia 2005;19:1613-20. 